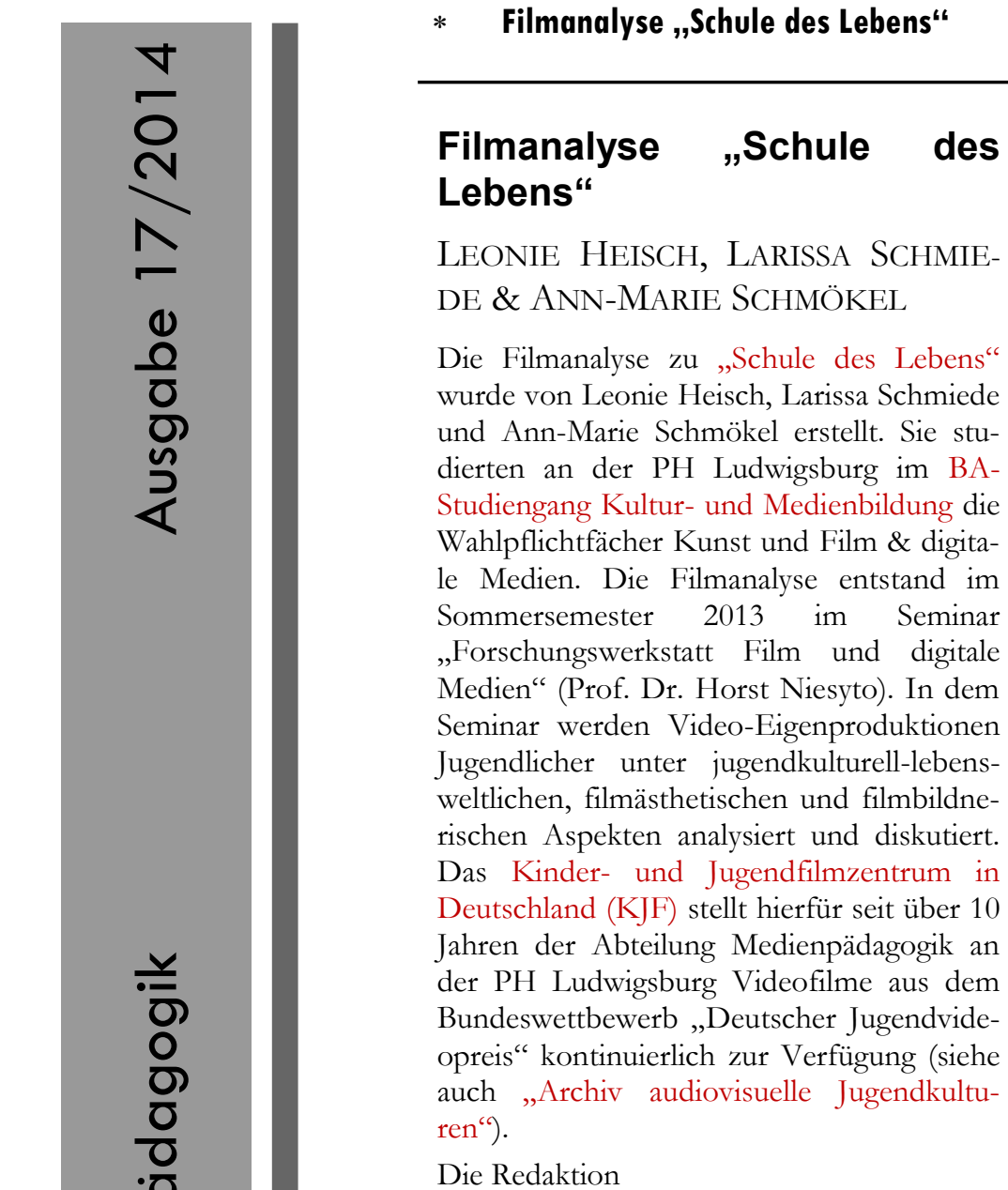

\section{Kurzbeschreibung der Filmstory}

Der Hauptcharakter Paul ist sieben Jahre alt und geht in die erste Klasse. Im Verlauf des Filmes philosophiert er über seinen weiteren Lebensweg und macht einen Entwurf aller Höhen und Tiefen, die er durchleben wird. Der Lebenslauf, von der Schulzeit über das Studium bis hin zu seiner späteren Arbeit als Arzt, aber auch zwischenmenschliche Aspekte, wie Familie, Freunde und vor allem seine, sich durch den ganzen Film ziehende, Liebe zu Katharina, werden in der „Schule des Lebens" reflektiert.

Paul wird eingeschult und hat nicht viele Freunde. Er verliebt sich in Katharina, das schönste Mädchen der Schule, welche allerdings nicht weiß, was Paul für sie empfindet. Die Schulzeit und die Pubertät durchlebt der Protagonist mit den typischen Problemen des Teenagerseins. Schlechte Noten und Pickel, genauso wie Mobbing in der Schule bleiben nicht aus. Nach dem Abitur zieht Paul raus in die große unbekannte
Stadt, weg von seinen mittlerweile getrennt lebenden Eltern, um Medizin zu studieren. Dort schließt er neue Freundschaften und lernt seine erste Freundin kennen, die allerdings nur zweckmäßig mit ihm zusammen ist. Neben Enttäuschungen in der Liebe und den üblichen Höhen und Tiefen, die ein junger Mensch zu bewältigen hat, stellt Paul fest, dass er immer noch in Katharina verliebt ist. In seiner Arbeit als Arzt erlebt Paul viele schöne, aber auch traurige Momente, die ihn schließlich als alten Mann, mit 72 Jahren, am Fenster rauchend über sein Leben nachdenken lassen.

Der Film ist durch animierte Grafiken dargestellt, die voller Symbole und ironischer Darstellungen stecken, die somit einen Kontrast zwischen Pauls kindlicher Welt und den doch ernsten und sehr reifen Erfahrungen und Gedanken über sein Leben darstellen. Erst am Ende des Films wird die grafische Darstellung aufgelöst und man sieht Paul in seinem Klassenzimmer sitzen. Er zeichnet sich, als alten Mann, wie er am Fenster steht und raucht und blickt zu einem Mädchen auf, welches Katharina darstellen soll. Die beiden lächeln sich an. Insgesamt ist der Film eine Auseinandersetzung junger Menschen mit der heutigen Welt, mit ihren Herausforderungen und Ängsten, aber auch mit ihren Träumen und Wünschen. Der Film hat eine Länge von 4:43 Minuten.

\section{Kontextinformationen}

Der Film wurde im 5. Semester an der Hochschule Anhalt, Fachbereich Design von den Studenten Patrick Paulin, Falko Tilgner und Michelle Günther produziert. Das Projekt war eine Semesterarbeit, die aber auf Grund der Länge und Vielfalt erst zu Anfang des darauffolgenden Semesters fertig gestellt werden konnte. Die Motivation für den Film entstand aus dem Willen, eine Botschaft $\mathrm{zu}$ vermitteln und zum Nachdenken anzuregen. Außerdem wollten die Studierenden ihre Animationskenntnisse weiterentwickeln. Die farbenfrohe und lebhafte Welt eines Kindes erschien ihnen dabei als perfekter Rahmen, sich gestalterisch in viele Richtungen ausprobieren zu können. Sie sind der Meinung, dass der Film zu einer weiteren und tieferen Bedeutungsebe- 
ne gelangte, indem sie Kontraste miteinander kombinierten: Das heißt, die Darstellung der unbeschwerten, bunten und auch fröhlichen Welt eines Kindes in einer erwachsenen und realistischen Art und Weise über das Leben zu erzählen. Der Inhalt beruht dabei auf den persönlichen Erfahrungen der Produzenten und fiktiven Geschichten.

Den Großteil des Filmes konnten sie in der Grafik frei erstellen, dennoch benötigten sie einen Jungen im Alter von sieben bis zehn Jahren, den sie für bestimmte Animationen (Rotoskopie-Technik) und die Schlussszene aufnehmen mussten und als Sprecher benötigten. Diesen Jungen fanden sie dann in dem Sohn einer ehemaligen Studentin. Für die weibliche Hauptrolle mussten sie länger suchen, fanden aber schließlich ein Mädchen, das so war, wie sie es sich vorgestellt hatten.

Ausgestrahlt wurde der Film beim Backup Festival in Weimar, im Olympiapark in München und beim Bundesfestival Video 2012, wo er dann auch mit dem Publikumspreis ausgezeichnet wurde. Des Weiteren lief der Film beim MDR in der Sendung „Unicato“.

\section{Bedeutungsanalyse}

Der Film spricht verschiedene Lebensthematiken und somit auch Lebensgefühle an, die im Folgenden genauer untersucht werden. In den Fokus der Analyse werden drei klar dargestellte Hauptthemenbereiche und damit verbundene Gefühle gestellt: Umwelt und Umfeld, Ausbildung und Liebe. Darüber hinaus bieten weitere dargestellte Lebensaspekte und damit verbundene Lebensgefühle eine Grundlage der Bedeutungsanalyse, auf die im Folgenden jedoch nicht eingegangen wird. Wo welche Thematik im Film aufgegriffen wird, ist durch die beigefügte Minutenangabe erkennbar.

\section{Umwelt und Umfeld}

Der Protagonist des Films, Paul, wird sich mit sechs Jahren das erste Mal seiner Umwelt bewusst und entwickelt das Gefühl, diese definieren und sich an sie erinnern zu können (0:05-0:12). Ihm wird bewusst, dass er nicht nur ein Teil einer geformten Gesellschaft ist, sondern dass er die Möglichkeit hat, diese auch selber zu formen. Als Akteur seines eigenen Lebens verdeutlicht er, dass nicht nur akademische, sondern vor allem soziale Kompetenzen eine fundamentale Funktion im Leben einnehmen. Ob man Freunde hat oder nicht, ist eine entscheidende, aber auch ungewisse Frage, die im Mittelpunkt seines ersten Schuljahres steht (0:24-0:30). Dort erfährt er, dass die Rolle als Mobbing-Opfer auch ein befriedigendes Gefühl auslösen kann. Er opfert sich für Lars, der von seinem Vater zu
Hause misshandelt wird, als AggressionsVentil, in dem er kleine Gewaltattacken von ihm auf dem Schulhof über sich ergehen lässt. Paul entwickelt eine Empathiefähigkeit und stellt Lars' Wohl unter das eigene und beweist eine soziale Reife (1:13-1:37).

Im Laufe der Ausbildungsjahre erfährt Paul, dass man durch erfolgreiche akademische Leistung schnell falsche Freunde bekommt, die einen für reine Selbstzwecke ausnutzen. Dieser ernüchternde Erkenntnisstand lässt auch die wunderschön ausgemalte Zukunftsblase mit seiner ersten Beziehung Tina platzen und enden (3:00-3:22).

Ausbildung

Mit sieben Jahren wird der Protagonist eingeschult und durchlebt folgend eine klassische akademische Laufbahn. Nachdem die Schule mit dem Abitur abgeschlossen wurde, folgt das Medizin-Studium, welches ihm einen Job als Arzt in der Notaufnahme sichert.

Mit der Einschulung wird Paul bewusst, dass die „Faulenzer-Jahre“ nun ein Ende haben werden und er mit einer Leistungserwartung konfrontiert wird, die er zu Beginn seiner schulischen Laufbahn nicht gut erfüllen kann $(0: 16-0: 21)$. Er ist zwar eher ein schlechter Schüler, schöpft aber Selbstvertrauen, dass sein Potenzial später zu mehr als schulisch gute Noten reichen wird. Paul äußert schon bereits mit acht Jahren, dass er glaubt, dass sein Leben mit Höhen und Tiefen gefüllt sein wird (1:01-1:11). Er fühlt sich aber in dieser Aussage von seinem (Erwachsenen-)Umfeld nicht ernst genommen, sondern eher von ihm stigmatisiert, dass doch ein Kind noch keine Ahnung vom Verlauf des Lebens hat (0:49_ 1:00).

Nicht nur der akademische Übergang von Schule zur Universität ist eine aufregende Erfahrung, auch der Umzug vom ländlichen Dorf in die Großstadt weckt Neugier, Freude, aber auch Ungewissheit in Paul (2:38-2:53). Die Loslösung aus dem Elternhaus, aus den festen, vertrauten Strukturen, und die Reise in einen neuen Lebensabschnitt, in die große neue noch undurchschaubare Stadt, nimmt Paul als eine Herausforderung an und meistert sie. Nach Abschluss des Studiums bekommt er einen Job in der Notaufnahme und erfährt dort schöne, aber auch traurige Momente (3:23-3:30). Der schmale Grad zwischen Trauer und Freude lässt ihn oft zweifeln, aber auch kämpfen, um einen guten Job als Arzt zu machen.

Liebe

Mit den Worten „Sie ist das schönste Mädchen der Schule" (0:36-0:38), umschreibt der Protagonist seine emotionale Zuneigung zu 
seinem Schul-Schwarm namens Katharina. Doch leider stößt diese Zuneigung nicht auf Gegenseitigkeit, denn Katharina ist zwar offen für Zuwendung männlicher Art, aber leider nicht für die von ihm (0:39-0:42). So erfährt Paul auf der einen Seite schon frühzeitig das Gefühl des Verliebtseins, jemanden mit besonderem Blick zu betrachten, jedoch muss er auf der anderen Seite die Ablehnung hinnehmen. Eine Ablehnung, die jedoch unausgesprochen ist, denn Paul legt seine Zuneigung Katharina gegenüber nicht offen dar, sondern geht davon aus, dass sie kein Interesse hat. Das Gefühl unglücklich und heimlich verliebt zu sein, stellt sich als eine schmerzhafte Erfahrung für Paul heraus, die er auch in den kommenden Schuljahren bis zur Pubertät leider spüren muss. Die imaginären, romantischen Zukunftsvorstellungen mit ihr bewähren sich auch im jugendlichen Alter nicht. Sie zieht ihm weiterhin andere Männer vor und stellt ihn vor die Sinnfrage: „Was bedeutet eigentlich schon Liebe? Manchmal Alles aber manchmal auch Nichts“ (1:50-2:08'). Denn nicht nur die enttäuschende Erfahrung mit Katharina und Tanja (siehe „Umwelt/Umfeld“), sondern auch die gescheiterte elterliche Liebe lassen ihn an dem Grundgerüst der Paarbeziehung zweifeln. Mitten in der Pubertät des Protagonisten beschließen die Eltern sich zu trennen, da der Vater die abwechslungsreichere Beziehung zu einer jüngeren Dame vorzieht (2:10-2:24). Paul übernimmt sehr viel Reife und Verantwortung, indem er für seine Mutter als emotionale Stütze da ist und sie im Alltag unterstützt (2:25-2:33). Womöglich stoßen, nach dem Empfinden des Protagonisten, zwei verschiedene Auffassungen von Liebe und der damit einhergehenden Paarbeziehung aufeinander. Seine Auffassung, der an dem romantischen Gefühl des einmaligen Verliebtseins in eine Frau festhält, und die seines Vaters, der Liebe einfach ersetzen zu scheinen kann. Eventuell nicht losgelöst von diesem innerlichen Konflikt lebt Paul sein Leben eher beruflich erfüllt weiter und reflektiert sein Leben mit 72 Jahren alleine, ohne eine weitere Partnerschaft eingegangen $\mathrm{zu} \operatorname{sein}(3: 37-3: 51)$.

Symbolik im Film

\section{$\underline{\mathrm{Uhr}(0: 10)}$}

Der Film startet mit der Vorstellung von Paul, als er sechs Jahre alt war. Hier wird ein Sprung über ein Jahr gemacht zu Pauls Einschulung. Die Zeiger der Uhr, die gezeigt werden, verlaufen im Schnelldurchlauf, somit soll durch die vielen vergangenen Stunden und Minuten das vergangene Jahr repräsentiert werden.

\section{Baum (0:14)}

Der Baum sieht aus, als wüchse er aus der Uhr heraus, und fängt langsam an, grüne Blätter zu bekommen. Als er blüht, fangen die grünen Blätter an, braun zu werden, die dann anschließend abfallen und vom Wind verweht werden. Diese Entwicklung läuft parallel zu den sich bewegenden Zeigern der Uhr ab, somit soll auch hier die schnell vergehende Zeit angesprochen werden. Die Blätter des Baumes sind Zeichen für die vier Jahreszeiten, die ein Jahr hat und die unterschiedlichen Phasen, die ein Baum während dieser Zeiten durchlebt, genauso wie Menschen auch.

\section{Anonymität der Mitschüler (0:23)}

Nachdem Paul eingeschult wurde, sieht man seine Klassenkameraden. Außer ihm sind alle Mitschüler anonymisiert, also nur als durch Umrisse dargestellte Körper angedeutet. Als Paul in die Schule kommt, möchte er neue Freundschaften schließen, was allerdings nicht so klappt, wie er sich das vorstellt. Dadurch, dass die Mitschüler alle gleich aussehen, stellen sie eine geschlossene Masse dar, die sich klar von Paul abgrenzt. Keiner von ihnen sticht heraus und freundet sich mit ihm an, keiner der Schüler wird also als besonders dargestellt, außer Katharina, das Mädchen, in das Paul verliebt ist. Sie ist genauso wie Paul selbst in realer, individueller Darstellung sichtbar.

\section{Träne (0:46)}

Die Träne, die Katharina verliert, soll deutlich machen, dass Paul hinter die Fassade ihrer Schönheit blickt und sie „so sieht, wie sie wirklich ist". Katharina wird als schönstes Mädchen der Schule betitelt, die von allem umworben wird. Im Gegensatz zu allen anderen ist Paul aber der Einzige, der sieht, dass mehr in Katharina steckt. Die Träne steht in diesem Fall also für die Emotionen und Gefühlsregungen, also ein Stück weit Katharinas Charakter, der für Paul eine genauso große Rolle spielt wie ihr Aussehen.

\section{Achterbahn mit Auto (0:50)}

Die Autobahn hat zweierlei Bedeutung in diesem Zusammenhang, da sie einerseits die Höhen und Tiefen des Lebens und andererseits eine für Pauls Alter charakteristische Spielkonsole symbolisieren soll. Paul spricht davon, dass ihm durchaus bewusst sei, dass sein Leben immer Höhen und Tiefen haben wird, also es Phasen gibt, in denen alles gut, aber auch Phasen, in denen Dinge schlecht laufen. Das Auto fährt auf dieser Achterbahn hoch und tief und durch Loopings und zeigt so eine „Achterbahn der Gefühle“, die jeder Mensch zeitweise durchläuft. Der zweite 
Aspekt ist der der Spielkonsole. Paul ist der Meinung, mit seinen Gefühlen nicht ernst genommen zu werden, da sich Jungs in seinem Alter mehr für Spielkonsolen interessieren als für wirklich wichtige Dinge. Die auftauchende Hand, die das Auto durch einen Joystick steuert, zeigt also das Klischee eines Konsolen-spielenden Jungens.

\section{Mathearbeit (1:06)}

Pauls schulische Probleme werden durch eine Mathearbeit symbolisiert. Mathe gilt für viele Schüler als klassisches Problemfach, womit sie sich schwer tun. Hier wird zwar gezeigt, dass Paul schlechte Noten in Mathe hat, er aber durchaus Potenzial in anderen Bereichen aufweist und es somit vollkommen menschlich und legitim ist, nicht alles zu können oder gut in allem zu sein.

\section{Mobbing/ Häusliche Gewalt (1:25)}

Paul wird in der Schule von einem Jungen gemobbt und verprügelt, der selbst von zu Hause aus Gewalt erfährt. In der Weise, in der der Junge Paul misshandelt, wird durch einen Rollentausch gezeigt, wie der Junge auf gleiche Weise von seinem Vater misshandelt wird. Die Gewalt in der Schule ist somit eine Verlagerung dessen, was dem Jungen zu Hause wiederfährt und eine Art das zu kompensieren, was einem selbst angetan wird.

\section{Spiegelszene (1:39)}

Auch hier läuft der Film im Schnelldurchlauf, um eine Entwicklung und Veränderung, die über längere Zeit geht, darzustellen. Paul sieht sich im Spiegel an und der Zuschauer sieht sein Spiegelbild, welches sich durch wechselnde Frisuren stetig verändert. Die Frisuren und die Pickel in Pauls Gesicht stehen für das Teenager sein. Paul experimentiert mit Frisuren, hat Hautprobleme und sein Körper fängt an, sich zu verändern. Die Pubertät, die hier dargestellt wird, bringt viele neue Erfahrungen und Veränderungen mit sich, die junge Menschen durchleben müssen.

\section{Wolken/ Himmel (2:01)}

Das Flugzeug am Himmel, welches mit Wolken das Wort „Liebe“ durch seinen Flug formt, ist ein Zeichen für die Liebe, die Paul nach wie vor für Katharina empfindet. Der Himmel, also das Himmlische, ist der Ort, an dem alles Gute herrscht und die Liebe immer siegt. Auch werden damit Träumereien dargestellt, der Himmel also als Ort der Träume, an dem Paul und Katharina zusammen sind.

\section{Tag/ Nacht (2:12)}

Hier wird immer wieder der gleiche Ablauf dargestellt: Der Vater verlässt morgens das Haus, um zur Arbeit zu gehen, und kommt abends wieder. Morgens geht die Sonne auf, wenn er abends zurückkommt, geht die Sonne unter. Hier wird ein Alltagstrott repräsentiert, dem die Eltern unterliegen. Die Ehe ist am Ende und besteht nur noch aus Gewohnheiten und den immer gleichbleibenden, wiederkehrenden Abläufen.

\section{Videospiel (2:29)}

Ein Super-Mario-Spiel funktioniert, indem man ein Level nach dem anderen löst und man so seinem Ziel Stück für Stück näher kommt. Dieses Spiel ist mit Pauls schulischer Laufbahn zu vergleichen. Das Endziel, das er erreichen will, ist das Abitur. Nach vielen Jahren und Herausforderungen, die Paul meistern musste, hat er das „Spiel“ Schule endlich geschafft und den „Endgegner“ Abitur besiegt.

Land (2:42)

Das ländliche Leben wird hier durch Berge, Kühe, Natur und Idylle dargestellt und steht somit im Kontrast zur Stadt. Das Landleben steht hier für Heimat, für einen Ort, an dem alles vertraut ist, an dem man nicht anonym ist, und ein Ort, dem Paul entfliehen will, um die Welt zu entdecken.

\section{$\underline{\text { Stadt (02:53) }}$}

Die Stadt wird durch Hochhäuser und urbanes Leben dargestellt. Es sind öffentliche Verkehrsmittel zu sehen, genauso wie Autobahnen, also eine ausgeprägte Infrastruktur als Symbol für Großstadt. Hierin sieht Paul etwas Unbekanntes und Neues, etwas, was voller Chancen steckt und einen neuen Lebensabschnitt symbolisiert. Großstadt bedeutet in diesem Fall also ein eigenständiges Leben, Studieren und Erwachsenwerden.

\section{Familie/ Hund/ Auto (03:13)}

Paul fängt an $\mathrm{zu}$ studieren und trifft dort seine erste Freundin, mit der er anfängt, $\mathrm{Zu}$ kunftspläne zu schmieden. Die Pläne für die Zukunft sind hier durch eine Familie mit Hund und einem Auto visualisiert worden. Diese Symbolik bedient sich des Klischees einer perfekten Familie und des Ziels, später einmal zu heiraten, Kinder zu bekommen und in guten finanziellen Verhältnissen zu leben. Es werden Statussymbole benutzt, die allgemeingültig anerkannt sind und als erstrebenswert gelten. Paul und seine Freundin malen sich also die ,perfekte“ Zukunft aus, die einem Bilderbuch entspringen könnte, und verdeutlichen so ihre Wünsche und Träume für die Zukunft.

\section{Herzrhythmusanzeige (03:26)}

Paul studiert Medizin und arbeitet in der Notaufnahme, wo er viele schöne, aber auch schreckliche Momente miterleben muss. Das Schlagen des Herzens, im Diagramm darge- 
stellt, bedeutet Leben und damit all das Schöne und Gute, was ein Arzt schaffen kann. Die Linie schlägt allerdings kurz danach nicht mehr aus, das Herz hört also auf zu schlagen und signalisiert damit den Tod. Mit dem Tod werden die Seiten an Pauls Arbeit als Arzt symbolisiert, die hart und schlimm sind. Da Leben und Tod unweigerlich zusammengehören und das ganze Leben voller guter und schlechter Tage steckt, wird hier ein Transfer zu den bereits angesprochenen Höhen und Tiefen, die ein jedes Leben mit sich bringt, hergestellt.

Rauchen am Fenster (3:39)

Das letzte Bild, welches zu sehen ist, zeigt Paul mit 72 Jahren, wie er rauchend an einem Fenster steht. Hier wird ein Mann gezeigt, der schon viel in seinem Leben durchlebt hat und auf sein Leben und Schaffen zurückblickt. Die Zigarette wirkt wie ein Angekommen-Sein, wie ein Genießen und zur Ruhe kommen. Paul steht allein am Fenster und blickt nach draußen, er ist in keinem schönen Haus mit Kindern und Ehefrau zu sehen, sondern wirkt leicht einsam. Dass er am Fenster steht, wirkt, als wäre er in Gedanken, als sehne er sich nach etwas oder als blicke er auf etwas zurück.

\section{Zusammenfassung}

Aufgrund der ästhetisch klaren Darstellung der einzelnen Thematiken wird dem $\mathrm{Zu}-$ schauer der Zugang zu diesen Themen erleichtert. Der hohe Stellenwert der Symbolik mit viel Ironie und teilweise übertriebener Darstellung führt zu einer intelligenten Reflexion typischer Lebensaufgaben. Durch die kindliche Erzählweise und das wiederkehrende Motiv des Spiels werden diese doch sehr schweren Lebensaufgaben vereinfacht aufgelöst. Dadurch wird eine positive Grundstimmung vermittelt, so dass der Zuschauer das Gefühl bekommt, schweren Zeiten in seinem Leben optimistisch und gestärkt entgegenschauen zu können. Der Film ist nicht nur eine Repräsentation von Lebensgefühlen, sondern beleuchtet ebenfalls kritisch das Nichternstnehmen von kindlichen Denkweisen. Die aufgeführten Aspekte mögen Gründe dafür sein, dass der Film den Publikumspreis beim Bundesfestival Video 2012 gewann. 